\title{
Waag, Philipp
}

\section{Digitalisierung als komplexer Gestaltungsspielraum. Eine systemtheoretische} Bestimmung disziplinärer und praktischer Herausforderungen in der

\section{Sozialen Arbeit}

Wunder, Maik [Hrsg.]: Digitalisierung und Soziale Arbeit. Transformationen und Herausforderungen. Bad Heilbrunn : Verlag Julius Klinkhardt 2021, S. 80-93

\section{Quellenangabe/ Reference:}

Waag, Philipp: Digitalisierung als komplexer Gestaltungsspielraum. Eine systemtheoretische Bestimmung disziplinärer und praktischer Herausforderungen in der Sozialen Arbeit - In: Wunder, Maik [Hrsg.]: Digitalisierung und Soziale Arbeit. Transformationen und Herausforderungen. Bad Heilbrunn : Verlag Julius Klinkhardt 2021, S. 80-93 - URN: urn:nbn:de:0111-pedocs-231621 - DOI: 10.25656/01:23162

https://nbn-resolving.org/urn:nbn:de:0111-pedocs-231621 https://doi.org/10.25656/01:23162

in Kooperation mit / in cooperation with:

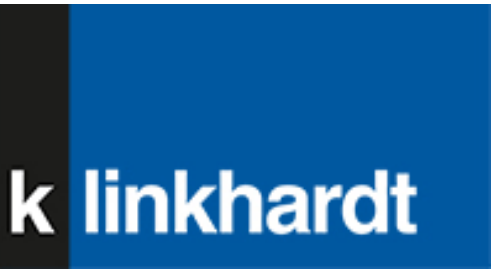

http://www.klinkhardt.de

\section{Nutzungsbedingungen}

Dieses Dokument steht unter folgender Creative Commons-Lizenz: http://creativecommons.org/licenses/by-nc-sa/4.0/deed.de - Sie dürfen das Werk bzw. den Inhalt unter folgenden Bedingungen vervielfältigen, verbreiten und öffentlich zugänglich machen sowie Abwandlungen und Bearbeitungen des Werkes bzw. Inhaltes anfertigen: Sie müssen den Namen des Autors/Rechteinhabers in der von ihm festgelegten Weise nennen. Dieses Werk bzw. der Inhalt darf nicht für kommerzielle Zwecke verwendet werden. Die neu entstandenen Werke bzw. Inhalte dürfen nur unter Verwendung von Lizenzbedingungen weitergegeben werden, die mit denen dieses Lizenzbedingungen weitergegeben werden,
Lizenzvertrages identisch oder vergleichbar sind

Mit der Verwendung dieses Dokuments erkennen Sie die Nutzungsbedingungen an.

\section{Terms of use}

This document is published under following Creative Commons-License: http://creativecommons.org/licenses/by-nc-sa/4.0/deed.en - You may copy, distribute and transmit, adapt or exhibit the work in the public and alter, transform or change this work as long as you attribute the work in the manner specified by the author or licensor. You are not allowed to make commercial use of the work. If you alter, transform, or change this work in any way, you may distribute the resulting work only under this or a comparable license.

By using this particular document, you accept the above-stated conditions of

\section{Kontakt / Contact:}

\section{peDOCS}

DIPF | Leibniz-Institut für Bildungsforschung und Bildungsinformation Informationszentrum (IZ) Bildung

E-Mail: pedocs@dipf.de

Internet: www.pedocs.de

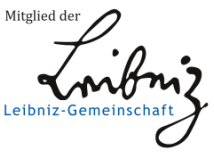




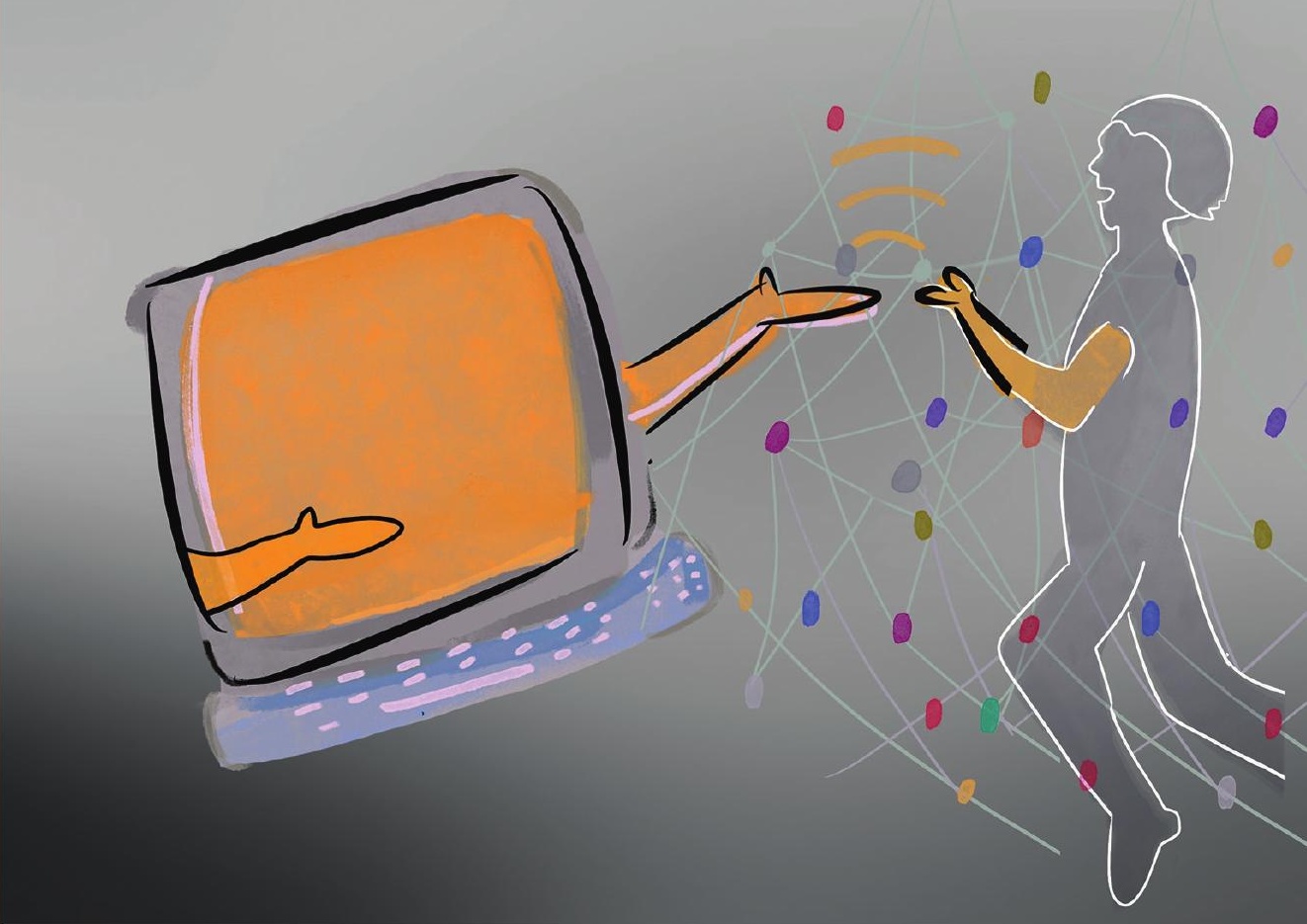

Maik Wunder (Hrsg.)

Digitalisierung und Soziale Arbeit

Transformationen und Herausforderungen 


\section{Maik Wunder \\ (Hrsg.)}

\section{Digitalisierung und Soziale Arbeit}

Transformationen und Herausforderungen 
Die Open Access-Publikation dieses Titels wurde durch Mittel des Forschungsschwerpunktes digitale_kultur der FernUniversität in Hagen finanziert.

Dieser Titel wurde in das Programm des Verlages mittels eines Peer-Review-Verfahrens aufgenommen. Für weitere Informationen siehe www.klinkhardt.de.

Bibliografische Information der Deutschen Nationalbibliothek

Die Deutsche Nationalbibliothek verzeichnet diese Publikation in der Deutschen Nationalbibliografie; detaillierte bibliografische Daten sind im Internet abrufbar über http://dnb.d-nb.de.

2021.h. () by Julius Klinkhardt.

Grafik Umschlagseite 1: (C) Miriam Tölgyesi (Rechte beim Herausgeber)

Druck und Bindung: AZ Druck und Datentechnik, Kempten.

Printed in Germany 2021.

Gedruckt auf chlorfrei gebleichtem alterungsbeständigem Papier.

Die Publikation (mit Ausnahme aller Fotos, Grafiken und Abbildungen) ist veröffentlicht unter der Creative Commons-Lizenz: CC BY-NC-SA 4.0 International https://creativecommons.org/licenses/by-nc-sa/4.0/

ISBN 978-3-7815-5911-0 digital doi.org/10.35468/5911

ISBN 978-3-7815-2473-6 print 


\title{
Inhaltsverzeichnis
}

\author{
Maik Wunder
}

Einleitung in den Band 9

\section{Digitalisierung und Veränderung Sozialer Arbeit}

\section{Udo Seelmeyer und Nadja Kutscher}

Zum Digitalisierungsdiskurs in der Sozialen Arbeit:

Befunde - Fragen - Perspektiven

\section{Maik Wunder}

Streiflichter durch Theorien zur Digitalisierung -

Digitalisierung als Gesellschafts-, Sozial- und Kulturtheorie

und deren mögliche Relevanz für die Soziale Arbeit

\section{Alexander Unger}

Digitalisierung oder Mediatisierung?

Ein analytischer Blick auf die Transformation

sozialpädagogischer Arbeitsfelder

Marc Witzel

Sozialpädagogische Orte im digitalen Raum

Philipp Waag

Digitalisierung als komplexer Gestaltungsspielraum:

Eine systemtheoretische Bestimmung disziplinärer und praktischer

Herausforderungen in der Sozialen Arbeit .80

\section{Digitalisierung und sozialarbeiterische Profession}

Fabian Hoose, Katrin Schneiders und Anna-Lena Schönauer

Von Robotern und Smartphones.

Stand und Akzeptanz der Digitalisierung im Sozialsektor

Anna-Sophie Brandt

Digitalisierung in der Gemeinwesenarbeit -

Bedarfe und Herausforderungen von Fachkräften in der Sozialen Arbeit 


\section{Diana Schneider}

Ein Schritt in Richtung De-Professionalisierung?

Plädoyer für eine intensive Diskussion über algorithmische Systeme in der professionellen Praxis

\section{Digitalisierung und Adressat*innen von Sozialer Arbeit}

Frieda Heinzelmann, Tanja Holzmeyer, Katrin Proschek und Frank Sowa

Digitalisierung als Projektionsfläche für Sehnsüchte und Ängste in Narrativen von wohnungslosen Menschen

Eva Maria Bäcker, Markus Grottke und Andreas König

Chancen digitaler Technologien für die Sozialen Arbeit?

Überlegungen zu Social Entrepreneurship unter Einsatz von digitalen

Lerntechnologien, virtuellem Kontext, New Work und

Selbstkontrollmechanismen

Anke Lang

Digitalisierung in der Kita - Bildung und Teilhabe für alle Kinder!

\section{Digitalisierung und sozialarbeiterische Ausbildung}

Martin Stummbaum und Kirsten Rusert

Zukünfte Sozialer Arbeit - digital und wie bei Ikea

Szenarien Sozialer Arbeit in der Digitalisierung

Joachim K. Rennstich

Neue Tricks für alte Hunde?

Digitalisierung als Herausforderung in Lehrvermittlung und Forschung

Jaqueline Veenker und Melanie Kubandt

Digitalisierung in Kindertagesstätten - Perspektiven von Lehrkräften an (Berufs-)Fachschulen für Sozialpädagogik 


\section{Digitalisierung und Forschung zu Sozialer Arbeit}

Angela Tillmann und André Weßel

Digitalisierung in der stationären Kinder- und Jugendhilfe zur Relevanz von digitalen Medien und Medienbildung in einem vernachlässigten Bildungskontext

Christian Ghanem, Markus Eckl, Robert Lehmann und Jean-Pierre Widerhold „Irgendwie fühle ich mich als Angehörige alleine gelassen“.

Eine automatisierte Analyse eines Onlineforums

für Angehörige von Inhaftierten

Almut Leh, Annabel Walz, Felix Engel und Matthias Hemmje

Historische Biografieforschung und Soziale Arbeit.

Interdisziplinäre Begegnungen im digitalen Raum .255 
80

Philipp Waag

\section{Digitalisierung als komplexer Gestaltungsspielraum: Eine systemtheoretische Bestimmung disziplinärer und praktischer Herausforderungen in der Sozialen Arbeit}

\section{Zusammenfassung}

Obwohl die Konstruktion und Adaption von Theorien der Sozialen Arbeit in der eigenen Disziplin und ihren Vorläufern auf eine lange Tradition blickt, wirkt ihre Theoriearbeit im Kontext der Digitalisierung bislang erstaunlich blass. Vielfach erfolgt die innerdisziplinäre Annäherung an Digitalisierungsphänomene vor allem als Interpretation fremdtheoretischer Zugänge. Dies erschwert einen selbstbewussten, primär an fachlichen Kriterien orientierten Digitalisierungsdiskurs und lässt schlichte Forderungen nach einer forcierten Digitalisierung in den Organisationen der Sozialen Arbeit ähnlich plausibel erscheinen wie ihre voreilige Ablehnung. Als externes Theorieangebot betont die soziologische Systemtheorie die Kontingenz der Techniknutzung ebenso wie jene des Technikverzichts und bestimmt die Digitalisierung in der Sozialen Arbeit damit als komplexen Gestaltungsspielraum für die organisierte Praxis.

\section{Einleitung}

„Und der Verdacht liegt dann nahe, daß die Semantik der Rationalität wie ein Singen und Pfeifen im Dunkeln praktiziert wird, um Unsicherheit und Angst zu vertreiben." (Luhmann 2019a, 329)

Im Anschluss an Bommes und Scherr (2012) versteht sich der vorliegende Text als Beitrag zu einer Soziologie der Sozialen Arbeit. Als soziologische Fremdbeschreibung bietet er im Kontext der Digitalisierung einerseits Anregungen für die Disziplin der Sozialen Arbeit, deren Theoriearbeit in Anbetracht der umfassenden gesellschaftlichen Wirkung der Digitalisierung bislang erstaunlich unbeteiligt wirkt (vgl. Kutscher 2020, 52). Andererseits empfiehlt er der organisationalen Praxis, Digitalisierung als ergebnisoffenen Gestaltungsspielraum zu bestimmen. Indem digitale und hybride Möglichkeiten der Leistungserbringung als Alternativen in den Blick geraten, wirken analoge Praktiken zunehmend kontingent und der Selektionszwang von Organisationen nimmt zu. Zwar entsteht dadurch grund- 
sätzlich die Möglichkeit, neben der Sinnhaftigkeit der Initiierung von Digitalisierungsvorhaben auch die Angemessenheit etablierter oder neuer analoger Praktiken $\mathrm{zu}$ verhandeln. Dieses Potenzial bleibt bislang jedoch ungenutzt, nicht zuletzt aufgrund der ausbleibenden reflexionstheoretischen Auseinandersetzung mit der Digitalisierung.

Anstatt Digitalisierung als umfassende Komplexitätssteigerung der Gesellschaft ernst zu nehmen, mit der es selbstbewusst umzugehen gilt, verkürzt sich der Diskurs innerhalb der Sozialen Arbeit allzu oft auf die Fragen: Technikeinsatz ja oder nein und wenn ja, dann wie? Die Forschungsförderung forciert diese Engführung, indem sie ausschließlich den Technikeinsatz fördert und der Sozialen Arbeit lediglich die Wahl des passenden Einsatzortes lässt (vgl. BMBF 2018). Der primäre Fokus von Forschungsvorhaben im Kontext der Digitalisierung liegt somit auf der Einführung und der Akzeptanzförderung technischer Innovationen (vgl. Hagen u.a. 2020). Gesellschaftliche Herausforderungen von Inklusions- und Exklusionsverhältnissen, für die sich die Soziale Arbeit primär interessiert (vgl. Bommes \& Scherr 2012), spielen lediglich eine sekundäre Rolle.

Die systemtheoretische Gesellschafts- und Organisationstheorie kann auf die „Komplexitätsvergessenheit“ (Nassehi 2018, 20)1 des bisherigen Digitalisierungsdiskurses in der Sozialen Arbeit aufmerksam machen und zugleich darauf hinweisen, dass analoge, digitale und hybride Möglichkeiten der professionellen Bearbeitung von Hilfsbedürftigkeit prinzipiell gleichermaßen kontingent erscheinen, sodass sich ihre fachliche Angemessenheit an disziplineigenen Kriterien messen lassen muss. Den fachlichen Sinn und Unsinn der Nutzung digitaler Technologien oder des Verzichts hierauf können die Organisationen der Sozialen Arbeit in ihren Entscheidungsprozessen aber nur dann ausreichend berücksichtigen, wenn ihnen die Disziplin grundlagentheoretische Kriterien zur Verfügung stellt. Andernfalls droht das notwendige Gerangel mit organisational zu berücksichtigenden Relevanzkriterien anderer gesellschaftlicher Teilbereiche (etwa jenen der Wirtschaft und der Politik) auf Kosten der Sozialen Arbeit auszubleiben.

\section{Reflexionstheorien und Fremdbeschreibungen gesellschaftlicher Teilsysteme}

Für die wissenschaftliche Beschreibung gesellschaftlicher Teilsysteme, wie der Politik, der Wirtschaft, des Rechts, der Erziehung, der Wissenschaft oder eben der

1 Unter Komplexitätsvergessenheit subsumiert Nassehi „solche Denkungsarten, die nach dem einen Hebel suchen, die einlinige Erklärungen abgeben, die die Einwirkungsmöglichkeiten auf die Gesellschaft überschätzen, die mit einem Grundprinzip arbeiten oder einen wünschenswerten Zustand der Welt kennen“ $(2018,20)$. 


\section{Philipp Waag}

sozialen Hilfe, ${ }^{2}$ sowie der Digitalisierung ${ }^{3}$ in diesen Systemen lassen sich grundlegend zwei Möglichkeiten unterscheiden: Die Teilsysteme der Gesellschaft beschreiben sich im Rahmen von Reflexionstheorien selbst oder sie werden aus Sicht einer wissenschaftlichen Disziplin, wie der Soziologie, fremdbeschrieben. ${ }^{4}$ Im Kontext von Luhmanns Systemtheorie hat André Kieserling (2004) diese Differenz besonders deutlich herausgearbeitet. Demnach orientieren sich Reflexionstheorien der Teilsysteme zwar an der Einhaltung wissenschaftlicher Kriterien, bleiben aber trotz des ,immensen Aufwand[s] an Tagungen und Zeitschriften, Lehrstühlen und Publikationen“ (Kieserling 2004, 73) „Theorien des Systems im System“ (Luhmann 1990a, 471). ${ }^{5}$ Dies wird daran deutlich, dass eine Reflexionstheorie die positive Selbsteinschätzung des Systems, das sie beschreibt, übernimmt, sich mit seinen Zielen und Institutionen identifiziert und dies zum Ausgangspunkt ihrer Selbstund Fremdbeobachtungen macht (vgl. Luhmann 1997, 965; Kieserling 2004, 5468). Eine Reflexionstheorie gilt als „Komponente der Selbstorganisation“ (Luhmann 2002, 202) eines Funktionssystems, als „Medium zur Identitätsstiftung“ (Domes \& Sagebiel 2016, 49). Auf diese Weise unterstützen Reflexionstheorien der Sozialen Arbeit die Praxis dabei, „die Komplexität von Lebensentwürfen, Situationen und Problemlagen, mit denen Sozialarbeitende konfrontiert sind, zu reduzieren" (Brielmaier 2019, 529).

Fremdbeschreibungen der systemtheoretischen Gesellschaftstheorie interessieren sich hingegen ausschließlich für die gesellschaftliche Funktion der verschiedenen

$2 \mathrm{Ob}$ Soziale Arbeit als eigenständiges Funktionssystem bestimmt werden kann, ist innerhalb der Systemtheorie strittig (vgl. Luhmann 1997, 633f.). Im vorliegenden Text wird Soziale Arbeit, anders als bei Bommes und Scherr (2012, 142ff.) oder bei Stichweh (2016, 68f.), als professioneller Kern eines Funktionssystems der sozialen Hilfe verstanden (vgl. zuerst Baecker 1994). Zur sozialen Hilfe (nicht aber zur professionellen Sozialen Arbeit) zählen zudem sekundäre Leistungsrollen (vgl. Stichweh 1988, 281ff.) in Form ehrenamtlicher Hilfen sowie soziale Alltagshilfen in Familien, Freundesund Bekanntenkreisen oder unter Fremden.

3 Der Digitalisierungsbegriff dient hier der Bezeichnung der vielfältigen kommunikativen Auswirkungen der zunehmenden Verwendung von Digitaltechnik, die analoge Werte erfasst und diese in digitale Daten umwandelt, sie speichern, übertragen und algorithmisch verarbeiten kann. Die Ergebnisse dieser Verarbeitungsprozesse reichen von der relativ simplen Übermittlung einer Nachricht an das Gerät eines Empfängers bis hin zu Big-Data-Analysen, deren Ergebnisse auch in der Sozialen Arbeit Entscheidungsfindungsprozesse verändern können (vgl. Schrödter u.a. 2020). Dabei kommt es nicht zur vollumfänglichen Digitalisierung der Gesellschaft, denn das würde den Verzicht auf analoge Formate bedeuten. Vielmehr digitalisieren sich einzelne Praktiken innerhalb der Gesellschaft, ohne dass analoge Möglichkeiten der Kommunikation dadurch verschwinden. Dies ebnet den Weg für hybride Formate, wie das Blended Counseling (vgl. Engelhardt \& Reindl 2016).

4 Im Sinne Thorngates (1976) gilt dabei, dass jede Theorie nur zwei von drei Kriterien erfüllen kann: allgemeine Anschlussfähigkeit, Genauigkeit und Einfachheit. „Je allgemeiner eine Theorie zu sein versucht, desto unvollständiger wird sie, je einfacher, desto falscher, und je genauer, desto unverständlicher" (Baecker 2016a, 119).

5 Soziologische Beschreibungen der Wissenschaft gelten demnach als interne Reflexionstheorien des Wissenschaftssystems (vgl. Kieserling 2004, 51). 
Teilsysteme, ohne diese normativ oder gar moralisch zu bewerten (vgl. Nassehi 2019a). Im Sinne der Tradition einer soziologischen Aufklärung, die die Funktionssysteme über sich selbst sowie über die Gesellschaft, in der sie sich befinden, informiert, kann der Mehrwert einer soziologischen Analyse für die Disziplin und die Praxis der Sozialen Arbeit ebenfalls darin bestehen, „die Komplexität der Welt [besser] zu erfassen und zu reduzieren" (Luhmann 1991a, 67; vgl. Bommes \& Scherr 2012, 22; Nassehi 2019b, 327). Als Beobachtung zweiter Ordnung tritt eine soziologische Beschreibung dabei in kritische Distanz zu ihrem Gegenstand, was in dem beschriebenen System allerdings Befremden und Ablehnung provozieren kann (vgl. z.B. Staub-Bernaconi 2000; Kieserling 2004, 62; von Stetten 2018). ${ }^{6}$

Eine soziologische Beobachtung der Digitalisierung in der Sozialen Arbeit, die sich außerhalb des beschriebenen Systems verortet, grenzt sich demnach explizit ab von reflexionstheoretischen Analysen, die Digitalisierung anhand sozialarbeiterischer Relevanzkriterien in den Blick nehmen (vgl. Bommes \& Scherr 2012, 42f.). Weder die eine noch die andere Perspektive gilt dabei als grundsätzlich besser, es handelt sich vielmehr um gleichwertige, aber andersartige Beobachterpositionen innerhalb einer polykontexturalen Gesellschaft (vgl. Luhmann 1990a, 634). Als Theorien des Systems im System sind Reflexionstheorien in ihren eigenen Systemen freilich immer anschlussfähiger als externe Fremdbeschreibungen.

\section{Soziale Arbeit und Digitalisierung: Reflexionstheoretische Flaute}

Im Hinblick auf die Digitalisierung in der Sozialen Arbeit fällt dann zunächst auf, dass neben den instrumentellen Bezügen zu Theorien aus anderen Disziplinen, etwa der Techniksoziologie (vgl. Eßer 2020), der Medienpädagogik (vgl. Hoffmann 2020) oder der Medienwissenschaft (vgl. Cleppien \& Hofman 2020), zumeist auch die Arbeit mit den eigenen Reflexionstheorien primär instrumentell erfolgt, insbesondere wenn sie der Rahmung empirischer Forschungsvorhaben dienen, in denen der Einsatz neuer Technologien gefördert werden soll (vgl. z.B. Lehmann \& Voit 2020, 57f.). Die selbstbewusste Positionierung der eigenen Reflexionstheorien im Digitalisierungsdiskurs (vgl. z.B. Tillmann 2020), ihre Adaption oder gar die Entwicklung neuer Theorien bleibt dagegen zumeist aus (vgl. Rösch 2019 für eine praxisfeldbezogene Ausnahme). Bezeichnend hierfür ist auch die überarbeitete Neuauflage der "Theorien der Sozialen Arbeit“ von Helmut Lambers

6 Darüber hinaus gibt es auch in allen anderen Teilsystemen Fremdbeschreibungen der jeweils anderen Systeme. Diese orientieren sich vor allem an den Leistungen, die das jeweilige System für sie zur Verfügung stellt, also ihrerseits an systemeigenen Relevanzkriterien (vgl. Kieserling 2004, 55). 
aus dem Herbst 2020, in deren Sachregister Begriffe wie „Computer“, „Internet“, „Digitalisierung“ oder „Mediatisierung“ weiterhin nicht auftauchen (vgl. Lambers 2020, 439ff.).

Während Beranek u.a. (2019) in ihrem Diskursüberblick Digitalisierung und Soziale Arbeit der „Digitalisierung aus der Perspektive von Sozialarbeitstheorien“ immerhin einen Abschnitt widmen (vgl. 231-235), ${ }^{7}$ endet die Suche im Lebrbuch Soziale Arbeit und Digitalisierung, abgesehen von wenigen professionstheoretischen Anmerkungen, indes vergeblich (vgl. Stüwe \& Ermel 2019). Selbst das umfangreiche Handbuch Soziale Arbeit und Digitalisierung von Kutscher u.a. (2020) eröffnet zwar „vielfältige theoretische und empirische Perspektiven auf Digitalisierungsprozesse“ (Kutscher u.a. 2020, 10) und widmet sich im ersten Kapitel den bereits erwähnten, primär fremddisziplinären Zugängen. Die Suche nach ausschließlich reflexionstheoretisch fokussierten Beiträgen endet für theorieinteressierte Leserinnen und Leser jedoch eher enttäuschend. Selbst im mit Forschung überschriebenen siebten Teil des Handbuchs finden sich lediglich zwei Methoden-Beiträge sowie ein Beitrag mit internationalen Bezügen.

Was Beranek u.a. für die Ausbildung angehender Fachkräfte fordern, könnte demnach auch der disziplinären Arbeit an und mit den eigenen Reflexionstheorien ins Gästebuch geschrieben werden: „die gesellschaftliche, kulturelle Breite der Digitalisierung [...] [sollte] in den Blick genommen werden“ $(2019,239)$. Nur so kann die Praxis der Sozialen Arbeit ein reflexionstheoretisch gefestigtes Selbstverständnis im Umgang mit den Herausforderungen der Digitalisierung entwickeln und sich wappnen für das Gerangel aus politischen, ökonomischen und fachlichen Anforderungen (vgl. Kreidenweis 2020, 129). Welche Impulse kann die systemtheoretische Gesellschafts- und Organisationstheorie hierfür bieten?

\section{Digitalisierung als organisationale Herausforderung}

Die gesellschaftliche Funktion der sozialen Hilfe besteht in der (Wieder-)Herstellung, Aufrechterhaltung und stellvertretenden Wahrnehmung der selbstbestimmten Teilhabe hilfsbedürftiger Individuen und Gruppen an der Gesellschaft (vgl. Bommes \& Scherr 2012, 144). Dabei ist die professionelle Bearbeitung von Hilfsbedürftigkeit durch die Soziale Arbeit immer eingebettet in Organisationen. Nur Organisationen können genügend Personen als Mitglieder rekrutieren, die zuverlässig und dauerhaft helfen und sich dabei organisationsspezifischen Anforderungen unterwerfen (vgl. Luhmann 1999, 36). Demnach sind es die Organisationen der Sozialen Arbeit, die darüber entscheiden, wie die Praxis mit der Digitalisierung umgeht und inwiefern sich im Prozess der Selbstveränderung (vgl. Muster \& Büch-

7 Dort beschränken sie sich allerdings, ohne dies explizit zu begründen, auf lediglich zwei Theorien. 
ner 2018, 258) auch die Leistungserbringung der Sozialen Arbeit sowie ihre organisationale Einbettung digitalisiert beziehungsweise hybridisiert (vgl. Seelmeyer \& Waag 2020). Welche Relevanz sozialarbeiterische, ökonomische, politische oder auch ethische Kriterien hierbei spielen, kann in jedem Digitalisierungsprojekt innerhalb der betroffenen Organisation im Rahmen ihrer Möglichkeiten prinzipiell selbst bestimmt werden.

Aus organisationaler Sicht bewirkt die Digitalisierung demnach zunächst die Steigerung der Komplexität ihrer gesellschaftlichen Umwelt und damit die Steigerung der Möglichkeiten eigener, sinnvoller Kommunikationen und Handlungen (vgl. Luhmann 1990b, 62; Baecker 2017, 5). ${ }^{8}$ Indem die Digitalisierung analoge Praktiken zunehmend kontingent erscheinen lässt und innerhalb von Organisationen ihrerseits nur als kontingentes Phänomen auftreten kann, forciert sie den Selektionszwang der Organisationen sowie ihr Entscheidungsrisiko, „angesichts einer unbekannten Zukunft nicht [zu] wissen, welche Selektion die richtige oder falsche ist" (Baecker 1998, 26; vgl. Luhmann 1984, 47). Weil Organisationen nicht über alle Informationen verfügen können, die ihnen bereits im Vorhinein Auskunft darüber geben würden, welche Entscheidung sich im Nachhinein als richtig erweist, müssen sie sich in der Gegenwart festlegen und für ihre Festlegungen Verantwortung übernehmen (vgl. von Foerster 1993, 73f.). ${ }^{9}$ Dabei gilt, dass Entscheidungen die Unsicherheit ihrer Ausgangslage nicht eliminieren, sondern lediglich absorbieren. Indem eine Organisation Unsicherheit in vorübergehende, mehr oder weniger verbindliche Sicherheit transformiert, kann sie weiterarbeiten. Dass dies immer durch Entscheidungen geschieht, „garantiert eine ständige Regenerierung von Unsicherheit" (Luhmann 2000, 216).

In diesem Sinne handelt es sich bei der Digitalisierung zunächst nicht um ein völlig neues Phänomen, denn die Absorption von Unsicherheit ist immer schon das Kerngeschäft von Organisationen (vgl. Luhmann 2000, 186), folglich auch von jenen der Sozialen Arbeit. Im Verhältnis zur gesellschaftlichen Umwelt lässt sich dies an ihrem Status als „Multireferenten“ (Wehrsig \& Tacke 1992, 229ff.) verdeutlichen. Organisationen wie Beratungseinrichtungen, soziale Dienste in Krankenhäusern und Kommunen, Jugendzentren, Werkstätten für Menschen mit

8 Dass die moderne Gesellschaft zwingend eine komplexe Gesellschaft ist, in der alle gesellschaftlichen Phänomene kontingent sind, berücksichtigen mitunter auch die Reflexionstheorien der Sozialen Arbeit (vgl. z.B. Kleve 2000; Kleve 2016). Für das Verständnis der Digitalisierung wurde dies aber - soweit ich es überblicken kann - noch nicht fruchtbar gemacht. Im Übrigen argumentiert Kleve zwar durchgehend systemtheoretisch, im Hinblick auf die Differenz von Selbst- und Fremdbeschreibung scheint er auf eine bewusste Verortung jedoch zu verzichten und primär dazu zu tendieren, soziologische Überlegungen in eine sozialarbeiterische Reflexionstheorie zu überführen, was freilich nur auf Kosten der soziologischen Genauigkeit zu haben ist.

9 „Damit ist zugleich gesagt, dass Nichtwissen nicht durch Wissen reduziert werden kann, sondern nur durch Entscheidungen“" (Luhmann 2000, 186). 
Behinderung, Wohnungslosenunterkünfte usw. können zwar in aller Regel hauptsächlich dem Teilsystem der sozialen Hilfe zugerechnet werden (vgl. Fußnote 2 auf S. 82), zugleich müssen sie bei ihrer Leistungserbringung aber die Kriterien weiterer Funktionssysteme berücksichtigen. So ist vor allem das Teilsystem der Wirtschaft mindestens sekundär an jeder Organisation beteiligt, die Lohn an ihre Mitglieder auszahlt, Räumlichkeiten mietet und Materialien anschafft (vgl. Luhmann 1997, 837; Luhmann 2000, 405f.). ${ }^{10}$ Ähnliches gilt für das Rechtssystem, da eine Organisation als „Rechtspersönlichkeit“ (Luhmann 2019b, 21) vertragliche Beziehungen eingehen kann und sich an geltende Gesetze halten muss (vgl. Teubner 1987). Zudem müssen die Organisationen der Sozialen Arbeit traditionellerweise die Anforderungen des politischen Systems und seines Wohlfahrtsstaates berücksichtigen (vgl. Bommes \& Scherr 2012, 172ff.). ${ }^{11}$ Somit befindet sich Soziale Arbeit immer schon in einem komplexen Geflecht aus verschiedenen, oft widersprüchlichen teilsystemischen Anforderungen, das weit über den prominenten Konflikt zwischen eigener Fachlichkeit und ökonomischer Rationalität der Dienstleistungserbringung hinausgeht (vgl. Stichweh 2016, 69f.). Ihre Reflexionstheorien können demnach nicht nur für ihre Fachkräfte in unmittelbaren Interaktionen mit Hilfsbedürftigen als komplexitätsreduzierende Leuchttürme fungieren, sondern prinzipiell auch jenen Organisationen Orientierung bieten, die die Soziale Arbeit in den unsicheren Gewässern der modernen Gesellschaft manövrieren und dabei immer auch an sich selbst denken (vgl. Baecker 2015, 10). ${ }^{12}$

\section{Möglichkeiten des organisationalen Umgangs mit neuartiger Unsicherheit $^{13}$}

Neu erscheint im Zuge der Digitalisierung nun das Ausmaß des Komplexitätsschubs, der die Gesellschaft insgesamt erfasst, ihre „bisherigen Institutionen, Konventionen und Routinen" (Baecker 2017, 5f.) herausfordert und darin laut Baecker nur mit der Einführung der Schrift und des Buchdrucks zu vergleichen sei (vgl.

10 Wenn eine Organisation der sozialen Hilfe nicht (mehr) staatlich alimentiert wird, sondern sich eigenständig refinanziert, ist sie ein (Sozial-)Unternehmen und damit primär eine Wirtschaftsorganisation (vgl. Kette 2019, 9).

11 Die Liste ließe sich um weitere teilsystemische Anforderungen ergänzen, etwa jenen der Religion (Caritas und Diakonie) und der Massenmedien (Öffentlichkeitsarbeit).

12 Dies schließt die Möglichkeit der Orientierung an verschiedenen Leuchttürmen mit widersprüchlichen Richtungsempfehlungen freilich nicht aus. (Reflexions-) Theorien sind ebenso kontingent wie alle anderen sozialen Phänomene (siehe auch Fußnote 3).

13 Hilfreiche Anmerkungen zu einer früheren Fassung dieses Abschnitts verdanke ich (in alphabetischer Reihenfolge) Diana Schneider, Konstantin Rink und Udo Seelmeyer. 
Baecker 2017, 5f.). ${ }^{14}$ Weniger einschneidend formulieren bereits Wehrsig und Tacke (1992, 232): „Indem die Informatisierung die Verfügbarkeit von Informationen steigert, [...] wächst für jeden einzelnen Akteur das Ausmaß genuiner Unsicherheit [...]."

Wenn die Diagnose stimmt, dass innerhalb der Sozialen Arbeit reflexionstheoretische Analysen fehlen, die diesen Komplexitätsschub angemessen berücksichtigen, drängt sich die Frage auf, woran sich die Organisationen und ihrer Mitglieder in der Praxis orientieren können, um die neuartige Unsicherheit im Zuge der Digitalisierung zu absorbieren.

Übliche Wege dürften in der Berücksichtigung von Wahrscheinlichkeiten und Gegenwartsdiagnosen liegen, die sich als Narrative durchsetzen und organisationale Entscheidungen plausibilisieren sowie als rationale Zweck-Mittel-Konstruktionen darstellbar machen (vgl. Luhmann 2019a, 320).

Während Wahrscheinlichkeiten als „operativ wirksame Fiktionen“ (Arlt \& Arlt 2020, 177) immer das Risiko ihres Nichteintreffens mitführen, selektieren Gegenwartsdiagnosen wie jene „der ,Digitalisierung ' [...] aus einer diffusen Wirklichkeit bestimmte Phänomene als signifikant, problematisieren diese als besonders relevant für die gesellschaftliche Zukunft und erzeugen auf diese Weise den Druck und die Motivation zu einem intervenierenden, die Zukunft gestaltenden Handeln“ (Alkemeyer \& Buschmann 2019, 119f.). Wahrscheinlichkeiten und Gegenwartsdiagnosen fungieren damit als handlungsleitende Narrative für die Gestaltung von Zukunft (vgl. Alkemeyer \& Buschmann 2019, 129). Systemeigene Beispiele hierfür sind etwa: „Die Sozialwirtschaft muss also zusehen, dass sie sich nicht aus der immer digitaler werdenden Mitte der Gesellschaft verabschiedet" (Kreidenweis 2020, 74), „Es wird darum gehen, Sozialarbeit und Sozialpädagogik in der Welt neu zu denken" (Pölzl \& Wächter 2019, 130), sowie: Der Technikschub der Sozialarbeit verweise ,höchstwahrscheinlich auf eine ziemlich radikale Umwandlung der Sozialarbeit selber" (Halfar 2018, 184).

Je stärker solche Narrative im innerdisziplinären und massenmedialen Diskurs verbreitet werden (vgl. Muster \& Büchner 2018, 253f.), desto stärker wird der Druck auf Organisationen, sich zwischen zwei dominanten Möglichkeiten zu entscheiden: Entweder so weiterzumachen wie bisher und dabei zu riskieren, als unzeitgemäß, weltfremd oder unbelehrbar kritisiert zu werden, ohne wissen zu können, ob diese Kritik berechtigt ist. Oder eigene Digitalisierungsmaßnahmen anzustoßen, obwohl zunächst unklar bleibt, ob sie sich aus fachlicher und organisationaler Sicht bewähren und mit welchen unintendierten Nebenfolgen sie einhergehen (vgl. Collingridge 1980; Gluckman \& Hanson 2019, 177ff.).

14 Baeckers These von einer nächsten Gesellschaft (vgl. 2007; 2018) und die Kritik an ihr (vgl. Luhmann 1997, 1143ff.; Holzer \& Fuhse 2010, 318ff.; Bommes \& Tacke 2011, 13f.; Nassehi 2019b, 184f.) soll hier keine Rolle spielen. 
Aus den vorangegangenen Überlegungen könnte sich für Organisationen der Sozialen Arbeit zusätzlich ein weiterer Weg des Umgangs mit der Digitalisierung ergeben, und zwar die prinzipielle Steigerung ihres Irritationspotenzials (vgl. Baecker 1998). Dies meint, „daß sie [...] zunächst einmal die Sensibilität in der Organisation für gesellschaftliche Probleme [bzw. Herausforderungen, PW] erhöhen [...], ohne von vornherein schon zu wissen, was daraus werden kann oder wie man sich, sei es innovativ, sei es eher konservativ, auf Irritationen dieses Typs einstellt" (Luhmann 2020, 507).

Auf diese Weise entstünde für Organisationen ein Gestaltungsspielraum, in dem sie die Komplexität der Digitalisierung nicht neutralisieren, sondern selbstbewusst im „Modus eines unverbindlichen Tuns als ob“ (Arlt \& Arlt 2020, 5) zelebrieren könnten (vgl. Miller-Kipp 2005; Esposito 2010, 165; Baecker 2016b, 154; Adamowsky 2018, 38f.). In Spielräumen gelten eigene „Gesetze und Gebräuche“ (Huizinga 2019, 21), innerhalb derer es möglich ist, „Disparates in versöhnende Formen [zu bringen]" (Adamowsky 2015, 17). Bestimmten Organisationen die Auseinandersetzung mit der Digitalisierung als ergebnisoffenen Gestaltungsspielraum, würden sie sich Möglichkeiten eröffnen, facheigene und fachfremde Anforderungen anhand organisationseigener Relevanzkriterien in Einklang zu bringen. Der Konflikt zwischen Mitgliedern, die zur reflexhaften Ablehnung technischer Neuerungen tendieren, und jenen, die Innovationen befürworten, könnte entschärft werden. Entscheidungen für oder gegen den Technikeinsatz stünden dann unter expliziten Revisionsvorbehalt und analoge, digitale sowie hybride Formen der Bearbeitung sozialer Hilfsbedürftigkeit könnten ergebnisoffen erprobt werden. ${ }^{15}$

Dass die Schaffung irritationssteigernder Spielräume im Umgang mit komplexen Herausforderungen nicht nur auf einer abstrakten, systemtheoretischen Ebene passend erscheint, zeigen neben Konzepten wie der Regulatory Sandbox (vgl. Hagen 2020) auch die vielen Reallabore, die es in den Bereichen der sozial-ökologischen Forschung und der politischen Partizipationsforschung gibt (vgl. Bischof u.a. 2020). Zwar gilt hier häufig die Prämisse der Entwicklung technischer Innovationen als Maßstab (vgl. BMWi 2019; Hagen 2020, 167f.). Deutet man entsprechende Konzepte und Methoden grundsätzlicher, können sie jedoch einen Rahmen für ergebnisoffene Spielräume bieten, in denen die gesellschaftliche Komplexität der Digitalisierung ernst genommen wird. Innerhalb von Organisationen böten derartige Spielräume zudem einen Ort für (pädagogisch begleitete) Prozesse,

15 Mit der vielbeschworenen Gamification (vgl. Deterding u.a. 2011) hätte diese Art des Spielens ebenso wenig zu tun wie mit Serious Games (vgl. Gotto 2013, 139). Beiden ist daran gelegen, „Ambiguität, Spontanität und Flexibilität [...] aus einer Situation heraus[zu] regeln“ (Adamowsky $2015,20)$ und bei den Spielerinnen und Spielern intendierte Verhaltensänderungen zu bewirken. Gibt es für Probleme lediglich eine legitime Lösung, mag der Einsatz solcher Methoden allerdings gerechtfertigt sein (vgl. Lehmann \& Voit 2020, 56). 
in denen die Auseinandersetzung mit der Digitalisierung in Rollen-, Theater- und angeleiteten Freispielen ebenso zulässig erscheint wie das Spielen mit Simulationen oder ein abgewandeltes Design Thinking, das neben dem Spielen mit Prototypen auch das Ausprobieren des Verzichts auf technische Neuerungen nicht scheut.

\section{Schluss}

Während in traditionellen Gesellschaften Neuerungen eher gefürchtet und als Abweichungen abgelehnt wurden, hat die moderne Gesellschaft in vielen Bereichen eine Präferenz für Neues entwickelt (vgl. Esposito 2016, 428; Luhmann 2020, 497ff.). Im Kontext der Digitalisierung gilt dies insbesondere für den Einsatz technischer Innovationen in allen gesellschaftlichen Teilbereichen. Dass die Praxis Sozialer Arbeit dabei oftmals zwischen Innovationspositivismus und reflexhafter Ablehnung zu schwanken scheint, dürfte auch daran liegen, dass ihre Disziplin bislang keine angemessenen reflexionstheoretischen Analysen der Digitalisierung erarbeitet hat.

Auch die hier vorgenommene systemtheoretische Bestimmung versteht sich als Fremdbeschreibung und damit als externes Theorieangebot, zu dem es sich innerhalb der Sozialen Arbeit zu positionieren gilt. Die Systemtheorie ist sich der begrenzten Anschlussfähigkeit ihrer Beobachtungen innerhalb einer polykontexturalen Gesellschaft bewusst und tut sich daher traditionellerweise schwer damit, anderen Funktionssystemen und ihren Organisationen konkrete Handlungsempfehlungen auszusprechen. Dessen ungeachtet unternimmt der vorliegende Text den Versuch, Digitalisierung in der Sozialen Arbeit als Herausforderung für die disziplinäre Theoriearbeit sowie das organisationale Entscheiden ihrer Praxis ernstzunehmen und für Letzteres vorzuschlagen, Digitalisierung als ergebnisoffenen Gestaltungsspielraum zu bestimmen. Die Entscheidung über die Angemessenheit dieses Vorschlags und seine versuchsweise Umsetzung kann nur innerhalb der Sozialen Arbeit getroffen werden.

\section{Quellenangaben}

Adamowsky, Natascha (2015): Spiel und Wissenschaftskultur - Eine Anleitung. In: Dies. (Hrsg.): „Die Vernunft ist mir noch nicht begegnet“. Zum konstitutiven Verhältnis von Spiel und Erkenntnis. Bielefeld: transcript, 11-30.

Adamowsky, Natascha (2018): Spiel/en. In: Feige, Daniel Martin; Ostritsch, Sebastian \& Rautzenberg, Markus (Hrsg.): Philosophie des Computerspiels. Theorie - Praxis - Ästhetik. Stuttgart: J. B. Metzler, 27-42.

Alkemeyer, Thomas \& Buschmann, Nikolaus (2019): Das Imaginäre der Praxis. Einsatzstellen für eine kritische Praxistheorie am Beispiel von Gegenwartsdiagnosen. In: ÖZS (Suppl 2) 44, 117-138.

Arlt, Fabian \& Arlt, Hans-Jürgen (2020): Spielen ist unwahrscheinlich. Eine Theorie der ludischen Akion. Wiesbaden: Springer VS. 


\section{Philipp Waag}

Baecker, Dirk (1994): Soziale Hilfe als Funktionssystem der Gesellschaft. In: Zeitschrift für Soziologie 23(2), 93-110.

Baecker, Dirk (1998): Einfache Komplexität. In: Ahlemeyer, Heinrich W. \& Königswieser, Roswita (Hrsg.): Komplexität Managen. Strategien, Konzepte und Fallbeispiele. Frankfurt/M. \& Wiesbaden: FAZ/Gabler, 21-50.

Baecker, Dirk (2007): Studien zur nächsten Gesellschaft. Frankfurt/M.: Suhrkamp.

Baecker, Dirk (2015): Postheroische Führung. Vom Rechnen mit Komplexität. Wiesbaden: Springer Gabler.

Baecker, Dirk (2016a): Theoriearbeit. In: Ders.: Wozu Theorie? Berlin: Suhrkamp, 115-133.

Baecker, Dirk (2016b): Das Spiel mit der Form. In: Ders. (Hrsg.): Probleme der Form, 2. Auflage. Frankfurt/M.: Suhrkamp, 148-158.

Baecker, Dirk (2017): Wie verändert die Digitalisierung unser Denken und unseren Umgang mit der Welt? Ausgangspunkte einer Theorie der Digitalisierung. In: Gläß, Rainer \& Leukert, Bernd (Hrsg.): Handel 4.0. Die Digitalisierung des Handels - Strategien, Technologien, Transformation. Wiesbaden: Springer Gabler, 3-23.

Baecker, Dirk (2018): 4.0 oder Die Lücke die der Rechner lässt. Leipzig: Merve.

Beranek, Angelika; Hill, Burkhard \& Sagebiel, Juliane Beate (2019): Digitalisierung und Soziale Arbeit - ein Diskursüberblick. In: Soziale Passagen 11(2), 225-242.

Bischof, Andreas; Freiermuth, Mira Mariane; Storz, Michael; Kurze, Albrecht \& Berger, Arne (2020): „Living Labs“ als Beispiel für die konzeptionellen Herausforderungen der Integration von Menschen in Technikentwicklung. In: Gransche, Brunso \& Manzesche, Arne (Hrsg.): Das geteilte Ganze. Horizonte Integrierter Forschung für künftige Mensch-Technik-Verhältnisse. Wiesbaden: Springer VS, 285-304.

Bommes, Michael \& Scherr, Albert (2012): Soziologie der Sozialen Arbeit. Eine Einführung in Formen und Funktionen organisierter Hilfe. 2., überarbeitete Auflage. Weinheim: Beltz Juventa.

Bommes, Michael \& Tacke, Veronika (2011): Einleitung. In: Dies. (Hrsg.): Netzwerke in der funktional differenzierten Gesellschaft. Wiesbaden: VS Verlag, 7-24.

Brielmaier, Julia (2019): Berufstätige Sozialarbeitende und die Theorien der Sozialen Arbeit - empirische Befunde. In: np 6/2019, 527-543.

BMBF (2018): Forschung an Fachhochschulen, FH Sozial. Online unter: https://www.forschung-fachhochschulen.de/bekanntmachungen/fh-sozial (Abrufdatum: 07.12.2020).

BMWi (2019): Freiräume für Innovationen. Das Handbuch für Reallabore. Online unter: https:// www.bmwi.de/Redaktion/DE/Publikationen/Digitale-Welt/handbuch-fuer-reallabore.pdf? blob=publicationFile (Abrufdatum: 07.12.2020).

Cleppien, Georg \& Hofman, Jana (2020): Ein sozialpädagogisches Remmidemmi mit Digitalisierung? In: Kutscher, Nadia; Ley, Thomas; Seelmeyer, Udo; Siller, Friederike; Tillmann, Angela \& Zorn, Isabel (Hrsg.): Handbuch Soziale Arbeit und Digitalisierung. Weinheim: Beltz Juventa, 58-73.

Collingridge, David (1980): The Social Control of Technology. New York: St. Martin's Press.

Detering, Sebastian; Dixon, Dan; Khaled, Rilla \& Nacke, Lennart (2011): From Game Design Elements to Gamefulness: Defining Gamification. In: Proceedings of the 15th international academic MindTrek conference: Envisioning future media environments. Tampere, 9-15.

Domes, Michael \& Sagebiel, Juliane (2016): Theorien als Medium zur Identitätsbildung - Die Bedeutung der Person des/der Lehrenden in der Vermittlung von Theorien der Sozialen Arbeit. In: Borrmann, Stefan; Spatscheck, Christian; Pankofer, Sabine; Sagebiel, Juliane \& Michel-Schwartze, Brigitta (Hrsg.): Die Wissenschaft Soziale Arbeit im Diskurs. Auseinandersetzungen mit den theoriebildenden Grundlagen Sozialer Arbeit. Opladen: Barbara Budrich, 49-69.

Engelhardt, Emily M. \& Reindl, Richard (2016): Blended Counseling - Beratungsform der Zukunft? In: Resonanzen. E-Journal für biopsychosoziale Dialoge in Psychotherapie, Supervision und Beratung, 4(2), 130-144. 
Esposito, Elena (2010): Ästhetik und Spiel. Formen der Kontingenz in der pluralen Realität. In: Pietraß, Manuela \& Funiok, Rüdiger (Hrsg.): Mensch und Medien. Philosophische und sozialwissenschaftliche Perspektiven. Wiesbaden: Springer VS, 159-177.

Esposito, Elena (2016): Pläne und die Zukunft. In: Rammert, Werner; Windeler, Arnold; Knoblauch, Hubert \& Hutter, Michael (Hrsg.): Innovationsgesellschaft heute. Perspektive, Felder und Fälle. Wiesbaden: Springer VS, 427-436.

Eßer, Florian (2020): Wissenschafts- und Technikforschung: Erklärungspotenziale für die Digitalisierung der Sozialen Arbeit. In: Kutscher, Nadia; Ley, Thomas; Seelmeyer, Udo; Siller, Friederike; Tillmann, Angela; Zorn, Isabel (Hrsg.): Handbuch Soziale Arbeit und Digitalisierung. Weinheim: Beltz Juventa, 18-29.

Foerster, Heinz von (1993): Ethik und Kybernetik zweiter Ordnung. In: Ders: KybernEthik. Berlin: Merve 164, 60-83.

Gluckman, Peter \& Hanson, Mark (2019): Ingenious: The Unintended Cost of Human Innovation. Harvard: Harvard University Press.

Gotto, Lisa (2013): Serious games. Einleitung. In: Freyermuth, Gudolf S.; Gotto, Lisa; Wallenfels, Fabian (Hrsg.): Serious Games, Exergames, Exerlearning. Zur Transmedialisierung und Gamification des Wissenstransfers. Bielefeld: transcript, 139-144.

Hagen, Julia (2020): Regulatory Sandboxes - Ein Instrument für digitale Innovationen im Gesundheitssektor. In: Pfannstiel, Mario A.; Kassel, Kristin \& Rasche, Christoph (Hrsg.): Innovationen und Innovationsmanagement im Gesundheitswesen. Technologien, Produkte und Dienstleistungen voranbringen. Wiesbaden: Springer Gabler, 163-180.

Hagen, Holger; Nitschke, Michél \& Gaugisch, Petra (2020): Die Lenkung des forschenden Blicks: Zur Funktion von Sozialwissenschaften und Philosophie in der Technikentwicklung. In: Kaminsky, Carmen; Seelmeyer, Udo; Siebert, Scarlet \& Werner, Petra (Hrsg.): Digitale Technologien zwischen Lenkung und Selbstermächtigung. Interdisziplinäre Perspektiven. Weinheim: Beltz Juventa, 153-184.

Halfar, Bernd (2018): Internet der Dinge: Sendung ohne Mouse. In: Kreidenweis, Helmut (Hrsg.): Digitaler Wandel in der Sozialwirtschaft. Baden-Baden: Nomos, 179-194.

Hoffmann, Bernward (2020): Medienpädagogik und Soziale Arbeit - kongruent, komplementär oder konträr im Umgang mit Digitalisierung und Mediatisierung. In: Kutscher, Nadia; Ley, Thomas; Seelmeyer, Udo; Siller, Friederike; Tillmann, Angela \& Zorn, Isabel (Hrsg.): Handbuch Soziale Arbeit und Digitalisierung. Weinheim: Beltz Juventa, 42-57.

Holzer, Boris \& Fuhse, Jan (2010): Netzwerke aus systemtheoretischer Perspektive. In: Stegbauer, Christian \& Häußling, Roger (Hrsg.): Handbuch Netzwerkforschung. Wiesbaden: VS Verlag, 313-324.

Huizinga, Johan (2019): Homo Ludens. Vom Ursprung der Kultur im Spiel, 26. Auflage. Reinbek: Rowohlt.

Kette, Sven (2019): Geld regiert die Welt? Facetten und Dynamiken der Ökonomisierung. In: Apelt, Maja; Bode, Ingo; Hasse, Raimund; Meyer, Uli; Groddeck, Victoria von; Wilkesmann, Maximiliane \& Windeler, Arnold (Hrsg.): Handbuch Organisationssoziologie. Wiesbaden: Springer, 1-20.

Kieserling, André (2004): Die Soziologie der Selbstbeschreibung: Über die Reflexionstheorien der Funktionssysteme und ihre Rezeption der soziologischen Theorie. In: Ders.: Selbstbeschreibung und Fremdbeschreibung. Beiträge zur Soziologie soziologischen Wissens. Frankfurt/M.: Suhrkamp, 46-108.

Kleve, Heiko (2000): Paradigmenwechsel in der Systemtheorie und postmoderne Sozialarbeit. In: Merten, Robert (Hrsg.): Systemtheorie Sozialer Arbeit. Neue Ansätze und veränderte Perspektiven. Opladen: Leske + Budrich, 47-66.

Kleve, Heiko (2016): Komplexität gestalten. Soziale Arbeit und Case-Management mit unsicheren Systemen. Heidelberg: Carl Auer. 


\section{Philipp Waag}

Kreidenweis, Helmut (2020): Sozialinformatik. Digitaler Wandel und IT-Einsatz in sozialen Organisationen, 3., überarbeitete Auflage. Baden-Baden: Nomos.

Kutscher, Nadia (2020): Perspektiven und Fragen für sozialpädagogische Forschung im Kontext einer digitalisierten Sozialen Arbeit. In: Bielefelder Arbeitsgruppe 8 (Hrsg.): Wie gehts weiter mit Forschung in der Sozialen Arbeit? Lahnstein: Verlag neue praxis, 45-60.

Kutscher, Nadia; Ley, Thomas; Seelmeyer, Udo; Siller, Friederike; Tillmann, Angela \& Zorn, Isabel (2020): Einleitung - Hintergrund und Zielsetzung des Handbuchs. In: Dies. (Hrsg.): Handbuch Soziale Arbeit und Digitalisierung. Weinheim: Beltz Juventa, 9-16.

Lambers, Helmut (2020): Theorien der Sozialen Arbeit. Ein Kompendium und Vergleich, 5., überarbeitete Auflage. Opladen: Budrich.

Lehmann, Robert \& Voit, Thomas (2020): Digitalisierung als Chance der Sozialen Arbeit - Ein Beispiel aus der Arbeit mit Menschen mit Behinderung. In: Kaminsky, Carmen; Seelmeyer, Udo; Siebert, Scarlet \& Werner, Petra (Hrsg.): Digitale Technologien zwischen Lenkung und Selbstermächtigung. Interdisziplinäre Perspektiven. Weinheim: Beltz Juventa, 55-75.

Luhmann, Niklas (1984): Soziale Systeme. Frankfurt/M.: Suhrkamp.

Luhmann, Niklas (1990a): Die Wissenschaft der Gesellschaft. Frankfurt/M.: Suhrkamp.

Luhmann, Niklas (1990b): Haltlose Komplexität. In: Ders.: Soziologische Aufklärung 5. Konstruktivistische Perspektiven. Opladen: Westdeutscher Verlag, 59-76.

Luhmann, Niklas (1991a): Soziologische Aufklärung. In: Ders.: Soziologische Aufklärung 1. Aufsätze zur Theorie Sozialer Systeme, 6. Auflage. Opladen: Westdeutscher Verlag, 66-91.

Luhmann, Niklas (1991b): Soziologie des Risikos. Berlin \& New York: Walter de Gruyter.

Luhmann, Niklas (1997): Die Gesellschaft der Gesellschaft. Frankfurt/M.: Suhrkamp.

Luhmann, Niklas (1999): Funktionen und Folgen formaler Organisation, 5. Auflage. Berlin: Duncker $\&$ Humblot.

Luhmann, Niklas (2000): Organisation und Entscheidung. Opladen \& Wiesbaden: Westdeutscher Verlag.

Luhmann, Niklas (2002): Das Erziehungssystem der Gesellschaft. Frankfurt/M.: Suhrkamp.

Luhmann, Niklas (2019a): Soziologische Aspekte des Entscheidungsverhaltens. In: Lukas, Ernst \& Tacke, Veronika (Hrsg.): Niklas Luhmann: Schriften zur Organisation 2. Theorie organisierter Sozialsysteme. Wiesbaden: Springer VS, 307-332.

Luhmann, Niklas (2019b): Überlegungen zum Verhältnis von Gesellschaftssystemen und Organisationssystemen. In: Lukas, Ernst \& Tacke, Veronika (Hrsg.): Niklas Luhmann: Schriften zur Organisation 2. Theorie organisierter Sozialsysteme. Wiesbaden: Springer VS, 3-10.

Luhmann, Niklas (2020): Sich im Undurchschaubaren bewegen. Zur Veränderungsdynamik hochentwickelter Gesellschaften. In: Lukas, Ernst \& Tacke, Veronika (Hrsg.): Niklas Luhmann: Schriften zur Organisation 4. Reform und Beratung. Wiesbaden: Springer VS, 497-509.

Miller-Kipp, Gisela (2005): Zufall, Selbstorganisation und Kreativität. Ein Versuch über ,die Natur des Spiels wider seine Pädagogisierung. In: Bilstein, Johannes; Winzen, Matthias \& Wolf, Christoph (Hrsg.): Anthropologie und Pädagogik des Spiels. Weinheim: Beltz, 273-291.

Muster, Judith \& Büchner, Stefanie (2018): Datafizierung und Organisation. In: Houben, Daniel \& Prietl, Bianca (Hrsg.): Datengesellschaft. Einsichten in die Datafizierung des Sozialen. Bielefeld: transcript, 253-277.

Nassehi, Armin (2018): Die letzte Stunde der Wahrheit. Kritik der komplexitätsvergessenen Vernunft, 2. Auflage. Hamburg: kursbuch.edition.

Nassehi, Armin (2019a): Rethinking Functionalism. Zur Empiriefähigkeit systemtheoretischer Soziologie. In: Kalthoff, Herbert; Hirschauer, Stefan \& Lindemann, Gesa (Hrsg.): Theoretische Empirie. Zur Relevanz qualitativer Forschung, 3. Auflage. Frankfurt/M.: Suhrkamp, 79-106.

Nassehi, Armin (2019b): Muster. Theorie der digitalen Gesellschaft. München: C. H. Beck. 
Pölzl, Alois \& Wächter, Bettina (2019): Digitale (R)Evolution in sozialen Unternehmen. Praxis-Kompass für Sozialmanagement und Soziale Arbeit. Regensburg: Walhalla.

Rösch, Eike (2019): Jugendarbeit in einem mediatisierten Umfeld. Impulse für ein theoretisches Konzept. Weinheim: Beltz Juventa.

Schrödter, Mark; Bastian, Pascal \& Taylor, Brian (2020): Risikodiagnostik und Big Data Analytics in der Sozialen Arbeit. In: Kutscher, Nadia; Ley, Thomas; Seelmeyer, Udo; Siller, Friederike; Tillmann, Angela \& Zorn, Isabel (Hrsg.): Handbuch Soziale Arbeit und Digitalisierung. Weinheim: Beltz Juventa, 255-264.

Seelmeyer, Udo \& Waag, Philipp (2020): Hybridisierung personenbezogener sozialer Dienstleistungen. In: Kutscher, Nadia; Ley, Thomas; Seelmeyer, Udo, Siller, Friederike; Tillmann, Angela $\&$ Zorn, Isabel (Hrsg.): Handbuch Soziale Arbeit und Digitalisierung. Weinheim: Beltz Juventa, 180-189.

Staub-Bernasconi, Silvia (2000): Machtblindheit und Machtvollkommenheit Luhmannscher Theorie. In: Merten, Robert (Hrsg.): Systemtheorie Sozialer Arbeit. Neue Ansätze und veränderte Perspektiven. Opladen: Leske + Budrich, 225-242.

Stetten, Moritz von (2018): Verfremdungsspiele. Zur Unterscheidung von vier Formen des systemtheoretischen Denkens. Weilerswist: Verbrück Wissenschaft.

Stichweh, Rudolf (1988): Inklusion in Funktionssysteme der modernen Gesellschaft. In: Mayntz, Renate; Rosewitz, Bernd; Schimank, Uwe \& Stichweh, Rudolf (Hrsg.): Differenzierung und Verselbstständigung. Zur Entwicklung gesellschaftlicher Teilsysteme. Frankfurt/M.: Campus, 261293.

Stichweh, Rudolf (2016): Die Soziologie und die Sozialarbeit. Die Profession der Inklusion in Gesellschaft. In: Ders.: Inklusion und Exklusion. Studien zur Gesellschaftstheorie. Bielefeld: transcript, 65-70.

Stüwe, Gerd \& Ermel, Nicole (2019): Lehrbuch Soziale Arbeit und Digitalisierung. Weinheim: Beltz Juventa.

Teubner, Günther (1987): Unternehmenskorporatismus. New Industrial Policy und das „Wesen“ der Juristischen Person. In: Kritische Vierteljahresschrift für Gesetzgebung und Rechtswissenschaft 2, 61-85.

Thorngate, Warren (1976): „In general“ vs. „it depends“: Some comments of the Gergen-Schlenker debate. In: Personality and Social Psychology Bulletin 2.4, 404-410.

Tillmann, Angela (2020): Veränderte Lebenswelten im Zuge gesellschaftlicher Digitalisierungsprozesse. In: Kutscher, Nadia; Ley, Thomas; Seelmeyer, Udo; Siller, Friederike; Tillmann, Angela \& Zorn, Isabel (Hrsg.): Handbuch Soziale Arbeit und Digitalisierung. Weinheim: Beltz Juventa, 89100.

Wehrsig, Christof \& Tacke, Veronika (1992): Funktionen und Folgen informatisierter Organisationen. In: Malsch, Thomas \& Mill, Ulrich (Hrsg.): ArBYTE. Modernisierung der Industriesoziologie? Berlin: Ed. Sigma, 219-239. 